\title{
Personalien
}

\section{Dr. Rolf Jansen verstorben}

https://doi.org/10.1515/iwp-2019-0006

In seinem 91. Lebensjahr ist am 17. November 2018 Dr. Rolf Jansen gestorben.

Als promovierter Chemiker war er seit 1960 bei der BASF in Ludwigshafen tätig. In den 1970er bis 1980er Jahren, einer Zeit, in der die Chemiedokumentation in Deutschland eine Blütezeit erlebte, verschrieb er sich der Dokumentation und leistete über seine Tätigkeit als Leiter der Dokumentationsstelle der BASF hinaus maßgebliche systematische Beiträge zur inhaltlichen Erschließung. Er war an der Erarbeitung des Thesaurus der Internationalen Dokumentationsgesellschaft für Chemie (IDC), einer von allen großen Unternehmen der chemischen Industrie gegründeten gemeinschaftlichen Einrichtung zur Auswertung und Indexierung von Fachliteratur der Chemie und ihrer Randgebiete beteiligt. Die IDC war eine GmbH zur Dokumentation der Chemieliteratur vor allem mit Methoden der elektronischen Datenverarbeitung sowie der Auswertung gespeicherter Information und ihrer Weitergabe insbesondere an die Gesellschafter. Gesellschafter waren u.a. die Firmen BASF, BAYER, Chemische Werke Hüls, Degussa, Dynamit Nobel, Henkel, Hoechst, Ruhrchemie, Wacker-Chemie u. a. In großen Firmen wird bis heute gerne auf die von der IDC eingespeicherten Bestände zugegriffen, auch wenn die IDC selbst liquidiert wurde und ihre Tätigkeit zum 31. Dezember 1993 beendete.

Ein Schwerpunkt der Tätigkeit von Rolf Jansen war neben seinen Aufgaben in der BASF die Normungsarbeit. 1977 übernahm er den Vorsitzend des Arbeitsausschusses Thesaurus und Indexierung NABD 4/5 des Normenausschusses Bibliotheks- und Dokumentationswesen im DIN. Unter seiner Ägide wurden die DIN 1463 Teil 1 und 2 (Thesaurus) sowie DIN 31623 Teil 1-3 (Indexierungsregeln) erarbeitet. Die neue Ausgabe DIN 1463 Teil 1 Erstellung und Weiterentwicklung von Thesauri: Einsprachige Thesauri erschien im September 1987, der Entwurf E DIN 1463 Teil 2
Erstellung und Weiterentwicklung von Thesauri: Mehrsprachige Thesauri im Dezember 1988. DIN 31623 Teil 1 bis 3 Indexierung zur inhaltlichen Erschließung von Dokumenten wurde im September 1988 veröffentlicht. Die Vorteile und Notwendigkeit der syntaktischen Indexierung stellte Rolf Jansen in einem Vortrag auf dem Deutschen Dokumentartag 1977 in Saarbrücken vor, dessen Textfassung in den NfD 29(1978)1, S. 19-23 abgedruckt ist.

Seinen Einfluss machte Rolf Jansen persönlich und durch den von ihm geleiteten Normungsausschuss auch bei der internationale Normungsarbeit für ISO 5964:1985 Guidelines for the establishment and development for multilingual thesauri und ISO 2788:1986 Guidelines for the establishment and development for monolingual thesauri durch eine aktive Mitarbeit geltend. Inzwischen hat die ISO-Norm ISO 259641 \& 2 über Thesauri, Vokabulare und andere Dokumentationssprachen die DIN 1463 offiziell abgelöst.

Rolf Jansen war über viele Jahre aktives Mitglied im DGD-Komitee Klassifikation und Thesaurusforschung (DGD-KTF). DGD-KTF und DIN-NABD 4/5 waren in Zusammensetzung und Aufgabenstellung praktisch spiegelbildlich. Zeitweise war er zudem Mitglied im Vorstand der DGD.

Seine Verbundenheit mit der Dokumentation demonstrierte Dr. Rolf Jansen nach außen u. a. durch seine E-MailAdresse dokufan@.... Er war seit 1963, also 55 Jahre lang Mitglied in der DGD/DGI und nahm an den Entwicklungen der Informationsverarbeitung mit wachem Interesse teil. So bezog er bis zuletzt diese Zeitschrift, die er auch regelmäßig las. Seine Arbeit wirkt in den Normen weiter, die nach wie vor Basis vieler aktueller Entwicklungen sind.

Der Vorstand der DGI übermittelt Frau Jansen sowie den Angehörigen von Dr. Rolf Jansen seine Anteilnahme. Wir werden unserem langjährigen Mitglied ein ehrendes Andenken bewahren.

Marlies Ockenfeld 


\section{Gmelin-Beilstein-Denkmünze für René Deplanque}

Die Gesellschaft Deutscher Chemiker (GDCh) hat Professor Dr. René Deplanque am 12. November 2018 auf der „14th German Conference on Chemoinformatics“, der Jahrestagung der GDCh-Fachgruppe „Computer in der Chemie“ in Mainz mit der Gmelin-Beilstein-Denkmünze ausgezeichnet. Die Silbermedaille, die mit einer Urkunde und einem Geldbetrag verbunden ist, wird von der GDCh an in- und ausländische Persönlichkeiten verliehen, die sich besondere Verdienste um die Geschichte der Chemie, die chemische Literatur oder die Chemie-Information erworben haben. Die GDCh würdigt René Deplanque für die Etablierung zahlreicher wichtiger Chemieinformationsdienste, unter anderem für die Weiterführung und Digitalisierung des „Gmelin“ (Gmelins Handbuch der anorganischen Chemie).

Der 1950 in Lübeck geborene Deplanque studierte an der Universität Hamburg Chemieingenieurwesen. Nach seinem Diplom erwarb er einen Master in Elektrochemie und wurde in Physikalischer Chemie am Sir John Cass College der London Guildhall University in Großbritannien promoviert. Nach einigen Jahre als Dozent an der Cambridge University und weiteren Jahren in der chemischen Industrie übernahm er 1988 bei der Max-Planck-Gesellschaft in Frankfurt am Main als Mitglied der Geschäftsleitung des Gmelin-Instituts die Gesamtprojektleitung der Digitalisierung des Gmelin-Handbuchs. Von 1994 bis 2011 leitete Deplanque zunächst als wissenschaftlicher, ab 2005 als alleiniger Geschäftsführer das anschließend liquidierte Fachinformationszentrum Chemie (FIZ CHEMIE) in Berlin. 2001 verlieh ihm die Technische Universität (TU) Berlin eine Honorarprofessur für Physikalische Chemie. Von 2012 bis 2014 war er als erster Deutscher Generalsekretär der internationalen Chemie-Vereinigung IUPAC. Gegenwärtig ist Deplanque Präsident der RD-Publisher, Berlin, und Geschäftsführer von INCOT.NET, Lübeck. 